Palabras clave: Gestos autolíticos deliberados, Adolescentes, Factores socio-culturales.

\title{
Gestos autolíticos deliberados (GAD) en adolescentes en el Oeste de Londres: Factores socio-culturales
}

\author{
Dinesh Bhugra, $\mathrm{PhD}$ \\ Neil Thompson* ${ }^{\star}$ \\ Jayshree Singh, PhD* \\ Elizabeth Fellow-Smith, MRCPsych** \\ * Institute of Psychiatry, P.O. Box 25, De \\ Crespigny Park, London SE5 8AF \\ ** Ealing Hammersmith \& Fulham Trust \\ Uxbridge Road Southall, Middlesex UB1 EU \\ UNITED KINGDOM
}

RESUMEN - Los estudios previos han sugerido que las tasas de gestos autolíticos deliberados (GAD) en adolescentes asiáticos y pertenecientes a otros grupos étnicos minoritarios no son diferentes de aquéllas del grupo mayoritario. En este estudio documentamos los factores socio-culturales implicados en los gestos autolíticos llevados a cabo por los adolescentes del oeste de Londres durante un período de un año.

Método. Se contactó con todos los casos de GAD en adolescentes para que participasen en el estudio. Empleando aproximaciones estándares y cualitativas, se recogieron los datos sobre los motivos del intento, identidad cultural y acontecimientos vitales.

Resultados. Se identificaron un total de 76 casos durante un año. En contra de lo esperado, las tasas de intento de suicidio fueron más bajas en los asiáticos que en los blancos. Los patrones de intento de suicidio mostraron que la sobredosis fue el sistema más comúnmente empleado y que el factor precipitante más común, sin tener en cuenta la etnia, fue el desacuerdo con los padres. Las sobredosis fueron, en ambos grupos, de tipo impulsivo. Los asiáticos documentaron con mayor frecuencia conflictos culturales, incluso aunque las tasas en los dos grupos no son diferentes.

Conclusiones. Cualquier estrategia preventiva debería incluir educación y ayuda para controlar el comportamiento impulsivo. 


\section{Introducción}

El gesto autolítico deliberado (GAD) no es un evento infrecuente entre adolescentes vulnerables al estrés, ya que están en el proceso de crear su individualidad y estableciendo sus identidades. Estos son tiempos de estrés para todos los adolescentes, pero especialmente para aquéllos pertenecientes a grupos étnicos minoritarios que viven en culturas mayoritarias, ya que pueden tener doble riesgo debido a su identidad cultural y étnica.

Existen varios problemas clave para identificar aquellos actos en los que se piensa que los individuos hayan cometido deliberadamente gestos autolíticos, lo que hace el reconocimiento y la evaluación de estos sujetos particularmente difíciles. Otro problema clave está en cuantificar el denominador, que es difícil de averiguar debido a la auto-atribución de la pertenencia a una etnia concreta. Las investigaciones previas ha mostrado datos discrepantes de tasas de intento de suicidio, lo que sugiere que hay problemas para determinar las tasas correctas. Además, las distintas áreas en el Reino Unido emplean distintas políticas para tratar a los niños y adolescentes que presentan sobredosis, lo que hace que un recuento preciso sea difícil, si no imposible.

En el Reino Unido, las tasas y la información referente a GAD en adolescentes de etnias minoritarias son bastante limitadas. McGibbon et al. (1992) documentaron que las tasas medias anuales de ingresos hospitalarios por sobredosis fueron parecidas en líneas generales en sus muestras de asiáticos y blancos, con un exceso de mujeres en ambos grupos, en Coventry. Goddard et al. (1996) documentaron, en el Sur de Londres, que las tasas de interconsulta entre los adolescentes negros por GAD fueron proporcionales al resto de la comunidad, sugiriendo que la etnicidad no se relaciona con tasas diferenciales, tal y como se ha documentado para los asiáticos con mayor edad, y especialmente en mujeres (Merrill y Owens 1986, Bhugra et al. 1999a). El riesgo de ideación suicida e intento de suicidio es mucho mayor en adolescentes que en controles de la comunidad (Bjarnsson y Thorlinsson 1994). Se ha documentado que este riesgo también es particularmente alto para los adolescentes hospitalizados por intentos de suicidio (Granboulon et al. 1995, Brent 1995). Kingsbury (1994) observó que los adolescentes asiáticos tenían menos probabilidad de ver a sus amigos y que confían incluso menos en ellos, y también consideraban a sus padres más controladores.

\section{Factores sociales y GAD}

Hay datos clínicos que sugieren que los niños y adolescentes que cometen deliberadamente gestos autolíticos tienen con más probabilidad una historia familiar de alcoholismo y conducta agresiva encubierta (Cohen-Sandler et al. 1982). Tanto los problemas con la familia, especialmente con los padres, como los problemas de relación, así como la intimidación en el colegio, contribuyen a las tasas de tentativa de suicidio (Hawton et al. 1996). Estos autores también documentaron ira, sentimientos de soledad y aislamiento, y no ser querido, como factores que contribuyen a este malestar y que conducen a cometer GAD.

Entre los adolescentes asiáticos, Biswas (1990) documentó que las mujeres tenían más probabilidad de describir sus intentos como accidentales. Los factores escolares (como el absentismo, la presión ejercida por el grupo, ser sujetos de intimidación y problemas con los profesores) y los factores domésticos (como conflictos familiares, violencia familiar, abuso de alcohol y abuso 
sexual en la familia) se han asociado con los intentos de suicidio (ver la revisión de Brent 1995); otro factor a considerar es el estrés social, que se ha dicho afecta a los adolescentes negros más que a los adolescentes blancos (Goddard et al. 1996).

Se ha mostrado que los problemas intergeneracionales son factores importantes en la génesis del malestar que lleva a los GAD, y tales conflictos son peores en culturas y grupos étnicos en los que un marco jerárquico masculino impone la sumisión a las mujeres. Handy et al. (1991) documentó que los conflictos intergeneracionales culturales fueron más significativos en los adolescentes asiáticos.

\section{Factores culturales y GAD}

Se ha dicho que los factores culturales juegan un papel importante en el comportamiento suicida. La mayoría de estudios de la diáspora india alrededor del mundo han demostrado tasas de GAD más altas que las esperadas, especialmente en mujeres (Maniam 1988, Mahy 1993), pero no se han documentado por separado las tasas entre los adolescentes indios. Las presiones culturales sobre las mujeres de la diáspora india se ha dicho que juegan un papel significativo en los GAD (Merrill y Owen 1986, Bhugra et al. 1999b). Por lo tanto, es más probable que alguno de estos factores afectará también a los adolescentes, especialmente si éstos están alcanzando un estado de individuación, y las presiones competitivas de los sujetos de su edad y de los padres pueden influir en sus comportamientos. Hawton et al. (1982) documentaron que 4/5 de los adolescentes que habían intentado suicidarse también documentaron incapacidad para comentar sus problemas con su padre, y casi la mitad describieron este problema en la relación con su madre.
Los objetivos del presente estudio fueron dobles: en primer lugar, establecer las tasas de asistencia por GAD en relación con la distribución étnica en un área con una gran población del Sur de Asia en el Oeste de Londres $y$, en segundo lugar, intentar identificar factores sociales y culturales que pueden contribuir a estas diferencias, si es que existen.

\section{Material y métodos}

\section{Muestra}

Los términos parasuicidio, intento de suicidio o GAD cubren un amplio rango de comportamientos y se refieren a cualquier acto deliberado con resultados no fatales que resulta en daño a uno mismo, y que incluye actos de ingestión masiva de sustancias muy por encima de lo que generalmente se considera como una dosis prescrita o terapéutica (Kreitman 1977). Utilizando esta definición, nos propusimos identificar todos los adolescentes que presentaran un GAD en el período de un año.

Los servicios infanto-juveniles del distrito de Ealing en el Oeste de Londres evalúan todos los casos de GAD en menores de 16 años, que por motivos de planificación son tratados por el equipo pediátrico. De este modo, se incluyeron todos los casos de GAD que se ponían en contacto con dichos servicios. Siguiendo las normas del equipo, los investigadores contactaron con sus padres para obtener su consentimiento informado, tras lo cual se entrevistó el adolescente y uno de los padres (generalmente la madre). En la mayoría de los casos, estas entrevistas tuvieron lugar en el hospital antes del alta. Cuando el especialista responsable o los padres rechazaron participar, se obtuvo información de la historia clínica. 
La etnicidad del individuo fue auto-atribuída, respetando así el consenso previo. En este contexto, los sudasiáticos son aquellos individuos cuyos padres, abuelos o ellos mismos son originarios del subcontinente indio.

\section{Evaluación}

La evaluación del GAD se realizó mediante una entrevista semiestructurada acerca de los detalles del intento (DACS-A); la versión para adultos había sido previamente utilizada en mujeres adultas con GAD (Bhugra et al. 1999a). Este cuestionario semiestructurado incluye un rango de preguntas hechas al paciente sobre el intento mismo, los factores precipitantes y perpetuantes, y los factores culturales y sociales. Además, se les dio a los adolescentes sudasiáticos el Asian Cultural Identity Schedule (ACIS, Cuestionario de Identidad Cultural Asiática) para medir la identidad cultural y el proceso de aculturación. El ACIS es una entrevista semiestructurada de 140 preguntas que cubre aspectos clave de la identidad cultural, incluyendo lenguaje, religión, actitudes, toma de decisiones, ocio y patrones de alimentación, etc. (Bhugra et al. 1999c). En todos los casos se administró también el Life Events Inventory (LEI, Inventario de Acontecimientos Vitales, (Cochrane y Robertson 1973), que fue modificado para incluir cuestiones extra sobre acontecimientos raciales.

En este artículo presentamos los datos sobre los detalles de la tentativa, así como algunos factores sociales y culturales.

\section{Resultados}

En total se identificaron 76 casos durante un año. De éstos 46 fueron blancos (38 mujeres, 8 varones, proporción 4,5:1), 15 sudasiáticos (12 mujeres, 3 varones, proporción 4:1), 6 de oriente medio (4 mujeres, 2 varones, proporción 2:1) y 9 mujeres negras (ningún varón negro). Las tasas de asistencia de los sudasiáticos y de los blancos han sido ya descritas (Bhugra et al., enviado para publicación). El grupo de sudasiáticos en este estudio incluye adolescentes indios, pakistaníes, de Bangladesh y de Sri Lanka.

Los intentos por sobredosis fueron un $66 \%$ de todos los casos, mientras que un $15 \%$ fueron por sobredosis y venodisección. El paracetamol fue la sustancia más comúnmente empleada en 28 casos (45\%), y en otros 9 casos (14\%) se utilizó paracetamol combinado con otras pastillas.

La sobredosis fue impulsiva en el $42 \%$ de los casos y planificada en el $16 \%$. Los sudasiáticos no refirieron haberse arrepentido de la tentativa, mientras que 25 mujeres blancas $(66 \%)$ y tres varones blancos $(25 \%)$ se arrepintieron de haber cometido las tentativas.

Los factores precipitantes se enumeran en la Tabla I y el factor más comúnmente identificado fue el desacuerdo con los padres. Más de la cuarta parte (29\%) reconocieron que el estar en desacuerdo con sus padres era un factor precipitante. La prevalencia de problemas de alcohol en casa fue baja. Estos datos se muestran en la Tabla II.

\section{Factores sociales y culturales}

Las variables de la entrevista semiestructurada relativas a la tentativa autolítica fueron divididas en áreas de problemas sociales (incluyendo problemas en el medio social), problemas en casa, problemas en el colegio y problemas emocionales. El tamaño de las submuestras es pequeño y, por tanto, sólo se presentan aquí los datos descriptivos, ya que la potencia estadística es limitada. 
Tabla I

Factores precipitantes (\%)

\begin{tabular}{|c|c|c|c|c|}
\hline Variable & $\begin{array}{l}\mathrm{N}^{\circ} \text { mujeres } \\
\text { blancas }\end{array}$ & $\begin{array}{c}\mathrm{N}^{\mathrm{o}} \text { varones } \\
\text { blancos }\end{array}$ & $\begin{array}{l}\mathrm{N}^{\mathrm{o}} \text { mujeres } \\
\text { asiáticas }\end{array}$ & $\begin{array}{c}\mathrm{N}^{\circ} \text { varones } \\
\text { asiáticos }\end{array}$ \\
\hline Discusión con los padres & $10(26,3)$ & $3(37,5)$ & $3(25)$ & $2(66,6)$ \\
\hline Discusión con amigos & $3(7,8)$ & $0(0)$ & $0(0)$ & $0(0)$ \\
\hline Pelea con amigo/hermano & $2(5,2)$ & $1(12,5)$ & $0(0)$ & $1(33)$ \\
\hline Ruptura con novio/a & $6(15,78)$ & $0(0)$ & $1(8,3)$ & $0(0)$ \\
\hline Problemas en el colegio & $2(5,2)$ & $0(0)$ & $1(8,3)$ & $0(0)$ \\
\hline Presión de los exámenes & $2(5,2)$ & $0(0)$ & $1(8,3)$ & $1(33,3)$ \\
\hline Medida disciplinaria en el colegio & $0(0)$ & $1(12,5)$ & $0(0)$ & $0(0)$ \\
\hline Problemas de alcohol en el hogar & $0(0)$ & $0(0)$ & $1(8,3)$ & $0(0)$ \\
\hline Ansiedad de separación & $2(5,2)$ & $2(25)$ & $0(0)$ & $0(0)$ \\
\hline
\end{tabular}

Se presentan datos descriptivos

\section{Problemas sociales}

Se muestran en la Tabla II. Las mujeres adolescentes mostraron con más frecuencia problemas de adaptación, comparadas con los varones. La quinta parte de las mujeres blancas y la cuarta parte de las mujeres sudasiáticas admitieron un consumo regular de alcohol. Los varones blancos mostraron problemas con sus compañeros, que se incrementaron gradualmente en los otros tres grupos.

\section{Problemas en el colegio}

Los adolescentes sudasiáticos faltaron a clase (absentismo) con mayor frecuencia y también tuvieron más problemas en el colegio. Se documentaron problemas en el colegio en un $45 \%$ de los sujetos. Éstos se muestran en la Tabla III.

\section{Ambiente familiar}

Más de la mitad del total de la muestra documentaron problemas de comunicación. Las adolescentes sudasiáticas declararon con mayor frecuencia conflictos culturales y generacionales y los padres de los niños asiáticos tienden a compararlos más con sus hermanos. Los factores implicados se muestran en la Tabla IV.

Tabla II

Problemas sociales (\%)

\begin{tabular}{lcccc} 
Variable & mujer blanca & varón blanco & mujer asiática & varón asiático \\
\hline Problemas de adaptación & $15(39,4)$ & $1(12,5)$ & $7(58,3)$ & $1(33,3)$ \\
Consumo de alcohol & $8(21,05)$ & $0(0)$ & $3(250)$ & $0(0)$ \\
Proceso legal & $10(26,0)$ & $1(12,5)$ & $2(16,6)$ & $0(0)$ \\
Actividad delictiva & $5(13,15)$ & $0(0)$ & $0(0)$ & $0(0)$ \\
Consumo de drogas & $9(23,68)$ & $2(25,0)$ & $3(25,0)$ & $2(66,6)$ \\
"Malas compañías" & $4(10,52)$ & $2(25,0)$ & $2(16,6)$ & $2(66,6)$ \\
Acosado/a & $5(13,15)$ & $1(12,5)$ & $0(0)$ & $0(0)$ \\
Problemas con compañeros & $18(47,13)$ & $3(37,5)$ & $5(41,66)$ & $3(100)$ \\
Problemas de relación & $23(60,5)$ & $6(75,0)$ & $9(75,0)$ & $3(100)$ \\
Sexualmente activo/a & $3(7,81)$ & $0(0)$ & $1(8,3)$ & $0(0)$ \\
Arrebato violento & $11(28,94)$ & $2(25,0)$ & $1(8,3)$ & $1(33,3)$ \\
\hline
\end{tabular}


Tabla III

Problemas en el colegio (\%)

\begin{tabular}{lcccc} 
Variable & $\begin{array}{c}\mathrm{N}^{\circ} \text { mujeres } \\
\text { blancas }\end{array}$ & $\begin{array}{c}\mathrm{N}^{\circ} \text { varones } \\
\text { blancos }\end{array}$ & $\begin{array}{c}\mathrm{N}^{\circ} \text { mujeres } \\
\text { asiáticas }\end{array}$ & $\begin{array}{c}\mathrm{N}^{\mathbf{o}} \text { varones } \\
\text { asiáticos }\end{array}$ \\
\hline Problemas académicos & $19(50)$ & $3(37,5)$ & $4(33,3)$ & $2(66,6)$ \\
Intimidado/a & $12(31,5)$ & $2(25,0)$ & $1(8,3)$ & $1(33,3)$ \\
Le gusta el colegio & $6(15,7)$ & $1(12,0)$ & $2(16,7)$ & $1(33,3)$ \\
Necesidades especiales & $8(21,05)$ & $2(25,0)$ & $0(0)$ & $0(0)$ \\
Le gustan los profesores & $0(0)$ & $0(0)$ & $4(33,3)$ & $0(0)$ \\
"Hace novillos" (absentismo escolar) & $6(15,7)$ & $1(12)$ & $3(25,0)$ & $2(66,6)$ \\
\hline
\end{tabular}

Tabla IV

Problemas domésticos (\%)

\begin{tabular}{lccccc} 
Variable & $\begin{array}{c}\mathrm{N}^{\mathrm{o}} \text { mujeres } \\
\text { blancas }\end{array}$ & $\begin{array}{c}\mathrm{N}^{\mathrm{o}} \text { varones } \\
\text { blancos }\end{array}$ & $\begin{array}{c}\mathrm{N}^{\mathrm{o}} \text { mujeres } \\
\text { asiáticas }\end{array}$ & $\begin{array}{c}\mathrm{N}^{\circ} \text { varones } \\
\text { asiáticos }\end{array}$ & $\mathrm{p}$ \\
\hline Fugas de casa & $9(23,68)$ & $1(12,5)$ & $4(33,3)$ & $0(0)$ & \\
Problemas de comportamiento & $10(26,3)$ & $2(25,0)$ & $4(33,3)$ & $2(66,6)$ & \\
Problemas de comunicación & $18(47,3)$ & $4(50,0)$ & $8(66,6)$ & $2(66,6)$ & \\
Comparación con sus hermanos & $2(5,2)$ & $0(0)$ & $4(33,3)$ & $1(33,3)$ & 0,008 \\
$\begin{array}{l}\text { Problemas de conducta } \\
\text { Conflicto cultural }\end{array}$ & $5(13,15)$ & $2(25,0)$ & $3(25,0)$ & $0(0)$ & \\
$\begin{array}{l}\text { Problemas económicos } \\
\text { Antecedentes de suicidio }\end{array}$ & $1(2,6)$ & $0(0)$ & $7(58,3)$ & $1(33,3)$ & 0,000 \\
$\quad 6(15,7)$ & $2(25,0)$ & $5(41,6)$ & $2(66,6)$ & \\
$\quad$ de los padres & $4(10,52)$ & $1(12,5)$ & $3(25,0)$ & $0(0,0)$ & \\
$\quad$ de los padrestes de enfermedad física & & & & & \\
$\begin{array}{l}\text { Conflicto generacional } \\
\text { Altas expectativas de los padres }\end{array}$ & $4(10,52)$ & $2(25,0)$ & $1(8,3)$ & $1(33,3)$ & \\
Abuso sexual & $4(10,5)$ & $2(25,0)$ & $7(58,8)$ & $2(66,6)$ & \\
\hline
\end{tabular}

\section{Problemas emocionales}

Siete adolescentes blancas (19\%) y dos sudasiáticas declararon alguna forma de trastorno de la conducta alimentaria, y el $60 \%$ de las mujeres blancas y el $42 \%$ de las asiáticas refirieron baja autoestima. Dos tercios de las mujeres sudasiáticas, todos los varones sudasiáticos, el 57\% de las mujeres blancas y el 33\% de los varones blancos comunicaron sentimientos de aislamiento. Éstos fueron síntomas auto-referidos y no diagnosticados clínicamente.
Casi la mitad de las mujeres blancas (42\%) reconocieron sentirse deprimidas, en comparación con el $37 \%$ de los varones blancos, el $16 \%$ de las mujeres sudasiáticas y todos los varones sudasiáticos. Dos mujeres blancas reconocieron rápidos cambios de humor.

Se llevó a cabo un modelo logístico lineal para examinar los efectos de la etnicidad y del género en la proporción de la muestra que experimentó problemas de conducta. Se observaron diferencias estadísticamente significativas sólo para los conflictos culturales y baja autoestima (gl: 3, 21,679 y 10,757, p $<0.05$, respectivamente). 


\section{Discusión}

Debemos señalar varias limitaciones de este estudio antes de interpretar los hallazgos. El tamaño muestral es pequeño y contiene un número desproporcionadamente grande de mujeres blancas. En segundo lugar, se debe tener presente la heterogeneidad de la etnicidad y de sus definiciones. En tercer lugar, puesto que tanto los numeradores como los denominadores son pequeños, la resulta difícil la generalización de los hallazgos. El grupo de los sudasiáticos está compuesto por una amplia y heterogénea colección de grupos étnicos. Sólo se presentan los datos descriptivos, puesto que los números son pequeños y, por tanto, las diferencias estadísticas son menos fiables.

Mediante la observación de todos los casos de GAD presentados en el hospital de Ealing durante el período de un año determinamos que las tasas obtenidas están relacionadas con la edad. Casi la mitad de las mujeres sudasiáticas menores de 16 años presentaron GAD, que es casi lo contrario de lo que ocurre en las mujeres sudasiáticas mayores de 16 años (Bhugra et al. 1999a).

El uso del paracetamol y otras pastillas como medio de intento de suicidio son hallazgos similares a los previamente documentados por Lockhart (1998), quien documentó que el $93 \%$ de los casos en el primer ciclo de su revisión y el $87 \%$ de los casos en el segundo ciclo de su revisión había tomado sobredosis y que las mujeres cometían más gestos autolíticos, un hallazgo confirmado en el presente estudio.

Ningún adolescente sudasiático declaró haberse arrepentido de su intento y la mayoría de los asiáticos que habían tomado una sobredosis lo hicieron de manera impulsiva, con un sentimiento de aislamiento. Esto sugiere que el aislamiento social y la sole- dad pueden provocar tentativas impulsivas, no sólo con mayor probabilidad y repetitividad, sino también posiblemente de manera más peligrosa.

\section{Factores sociales}

Los datos apoyan las observaciones previas de que una variedad de conductas descarriadas ("deviant") de la juventud se asocian con comportamientos de GAD. Los adolescentes en nuestra muestra estuvieron involucrados en actividades delictivas, consumo de alcohol y drogas y tuvieron problemas de adaptación y con sus compañeros.

Casi la mitad de la muestra refirió problemas en el colegio y otros problemas, tales como la presión ejercida por sus compañeros, acciones disciplinarias y la presión de los exámenes, fueron identificados como causas que contribuían a estas conductas. La intimidación fue prevalente de manera similar en ambos grupos, lo que sugiere que la etnicidad no fue una posible causa de intimidación. Una proporción mayor de adolescentes sudasiáticos declararon absentismo escolar, y esto podría reflejar su forma de evitar cualquier confrontación o conflicto. Esto debe ser estudiado en el futuro.

\section{Problemas en el hogar $y$ emocionales}

Estar en desacuerdo con alguien fue un factor precipitante común en los GAD. El desacuerdo entre amigos fue lo más común para las mujeres, especialmente con sus novios. Sin embargo, a menudo el desacuerdo fue una discusión con los padres. Fugarse de casa, tener problemas comportamentales de comunicación, un sentimiento de aislamiento y soledad y factores culturales pueden influir más a los sudasiáticos, como confirman los 
análisis logístico-lineales. Así, la baja autoestima, el aislamiento y el sentimiento depresivo sugieren que, en general, un sentimiento de desesperación puede ser prevalente, lo que añadido a desacuerdos y discusiones puede llevar a gestos autolíticos impulsivos. Las mujeres sudasiáticas deben hacer frente a más problemas en casa, tienen menos problemas sociales y en el colegio, tienden a consumir alcohol y drogas y refieren más aislamiento social; todo ello sugiere una interacción compleja de varios factores de vulnerabilidad.

Una proporción mayor de sudasiáticos (38\%) refirieron altas expectativas parentales, comparado con sólo un $12 \%$ de adolescentes blancos. Este estado crónico de disforia puede estar asociado a desacuerdos adicionales. A pesar del hecho de que las tasas de gestos autolíticos en el grupo de sudasiáticos y de blancos es similar, hay una mayor proporción en los primeros de conflictos culturales y baja autoestima. Éstos fueron también frecuentes en las adolescentes asiáticas de mayor edad, lo que sugiere que los conflictos culturales tienen una asociación clave y quizá longitudinal con los patrones de gesto autolítico. Las investigaciones longitudinales están indicadas para valorar cómo se resuelven dichos conflictos culturales, si es que esto sucede.

\section{Conclusiones}

Las tasas de intento de suicidio no son distintas en los dos grupos. Sin embargo, hay diferencias claras sociales y culturales que necesitan ser estudiadas en detalle en estudios multicéntricos con medidas cualitativas y cuantitativas detalladas. Se necesita valorar también las visiones parentales del conflicto. Es probable que los conflictos culturales aumenten conforme los individuos se hacen mayores. También es posible que dichos conflictos disminuyan en la próxima generación y en los adolescentes actuales.

\section{Agradecimientos}

Este estudio fue financiado por la North Thames R. \& D. y estamos agradecidos por su apoyo. Queremos mostrar nuestro agradecimiento también a todos los participantes en el estudio y a sus cuidadores, así como a los especialistas que nos permitieron acceder a sus pacientes.

\section{Bibliografía}

BHUGRA, D., DESAI, M., BALDWIN, D. Attempted Suicide In West London I. Rates Across Ethnic Communities. Psychological Medicine 29, 1125-1130, 1999.

BHUGRA, D., BALDWIN, D., DESAI, M., JACOBS, K.S. Attempted Suicide In West London II. Social And Cultural Factors. Psychological Medicine 29, 1131-1135, 1999.

BHUGRA, D., BHUI, K., MALLET, R., DESAI, M., SINGH, J., LEFF, J. Cultural Identity And Its Measurement: A Questionnaire For Asians. International Review Of Psychiatry 11, 244-249, 1999.

BHUGRA, D., BHUI, K., DESAI, M., SINGH, J., BALDWIN, D. The Asian Cultural Identity Schedule (ACIS): An Investigation Of Culture And Deliberate SelfHarm. International Journal Of Methods In Psychiatric Research 8, 212-218, 1999.

BISWAS, S. Ethnic Differences In Self-Poisoning: A Comparative Study Between An Asian And White Adolescent Groups. Journal Of Adolescence 13, 189-193, 1990.

BJARNSSON, T., THORLINSSON, T. Manifest Predictors Of Past Suicide Attempts In A Population Of Icelandic Adolescents. Suicide And Life Threatening Behaviour 24, 350-358, 1994.

BRENT, D.A. Risk Factors For Adolescent Suicide And Suicidal Behaviour: Mental And Substance Abuse Disor- 
ders, Family Environment Factors And Life Stress. Suicide And Life Threatening Behaviour 25 (Suppl) 52-63, 1995.

COCHRANE, R., ROBERTSON, A. The Life Events Inventory: A Measure Of The Relative Sensitivity Of Psychosocial Stressors. Journal Of Psychosomatic Research 17, 135-139, 1973.

COHEN-SANDLER, R., BERMAN, A., KING, R.A. Life Stress And Symptomatology: Determinants Of Suicidal Behaviour In Children. Journal Of American Academy Of Child And Adolescent Psychiatry 21, 178-186, 1982.

GODDARD, N., SUBOTSKY, F., FROMBONNE, E. Ethnicity And Adolescent Deliberate Self-Harm. Journal Of Adolescence 19, 513-521, 1996.

GRANBOULON, V., RABAIN, D., BASQUIN, M. The Outcome Of Adolescent Suicide Attempts. Acta Psychiatrica Scandinavica 91, 265-270, 1995.

HANDY, S., CHITTIRAMOHAN, R., BALLARD, C., SILVEIRA, W. Ethnic Differences In Adolescent Self-Poisoning: A Comparison Of Asian And Caucasian Groups. Journal Of Adolescence 14, 157-162, 1991.

HAWTON, K., FAGG, J., SIMKIN, S. Deliberate SelfPoisoning And Self Injury In Children And Adolescents Under 16 Years Of Age In Oxford 1976-1993. British Journal Of Psychiatry 69, 202-208, 1996.
HAWTON, K., COLE, D., O'GRADY, J., OSBORN, M. Motivational Aspects Of Deliberate Self-Poisoning In Adolescents. British Journal Of Psychiatry 141, 286-291, 1982.

KINGSBURY, S. The Psychological And Social Characteristics Of Asian Adolescent Overdose. Journal Of Adolescence 17, 131-135, 1994.

KREITMAN, N. Parasuicide. London. John Wiley, 1977.

LOCKHART, E. Audit Cycle On Young People Presenting With Self-Harm. Psychiatric Bulletin 22, 100-101, 1998.

MAHY, G. Suicidal Behaviour In The Caribbean. International Review Of Psychiatry 5, 261-269, 1993.

MANIAM, T. Suicide And Parasuicide In A Hill Resort In Malaysia. British Journal Of Psychiatry 149, 265-273, 1988.

McGIBBON, L., BALLARD, C., HANDY, S., MOHAN, R., SILVEIRA, W. Deliberate Self Poisoning In Asian And Caucasion 12 - 15 Year Olds. British Journal Of Psychiatry 161, 101-112, 1992.

MERRILL, J., OWEN, J. Ethnic Differences In SelfPoisoning - A Comparison Of Asian And White Groups. British Journal Of Psychiatry 148, 708-712, 1986. 